\title{
Familial progressive aphasia: its relationship to other forms of lobar atrophy
}

\author{
D Neary, J S Snowden, D M A Mann
}

\begin{abstract}
Two brothers presented with slowly progressive aphasia. One brother, who became behaviourally disturbed only at the end of his illness, was found at necropsy to have predominant left frontotemporal atrophy. The other brother developed severe behavioural disturbances shortly after the onset of language impairment. His brain revealed bilateral frontotemporal atrophy. In both there was non-Alzheimer's disease pathology with the histological features of loss of large cortical nerve cells, spongiform change and mild gliosis. The differential anatomical atrophy supports the view that clinical manifestations of lobar atrophy are dictated by the topographical distribution of a common underlying pathology, linking the syndromes of progressive aphasia to dementia of frontal lobe type (DFT) and DFT with motor neuron disease.
\end{abstract}

$(\mathcal{F}$ Neurol Neurosurg Psychiatry 1993;56:1122-1125)

Reports of patients with a slowly progressive aphasic disorder in association with focal cerebral atrophy have proliferated and it has become evident that a variety of language presentations may occur. However, patients with differing language presentations may have a similar underlying pathology. ${ }^{1}$ Moreover, affinities have been drawn between the pathological changes in progressive aphasia (PA) and those of dementia of frontal lobe type (DFT) ${ }^{2}$ and DFT with motor neuron disease. ${ }^{3}$ Accordingly, it has been suggested that the clinical syndromes of PA and DFT share a common pathological substrate, which differs only in the distribution of pathology within the frontotemporal lobes. The opportunity has arisen to address this issue more directly. Among the group of patients presenting with PA reported previously $^{1}$ two were brothers. Both shared a nonfluent pattern of language disorder, in the absence of perceptual and spatial difficulties. However, they differed with respect to development of behavioural symptomatology akin to that of DFT. This difference was reflected in differences in single photon emission computerised tomography (SPECT) and necropsy findings of the distribution of pathology within the frontal and temporal lobes. The patients' case histories are presented and discussed in the light of imaging and pathological findings.

\section{Case 1}

A right-handed carpenter, at the age of 62 , developed increasing difficulty with verbal expression and word finding. He made phonemic paraphasic errors in speech and in reading. His comprehension was regarded as normal. He became frustrated by his language disorder into which he retained full insight. His character was unchanged. He displayed initiative and continued to be skilled in carpentry. His language became progressively non-fluent and telegrammatic, with frequent literal but no verbal paraphasias. Eight years after the onset of symptoms he became mute and his comprehension profoundly impaired.

Significant behavioural alteration only became apparent 7 years after onset of his language disorder. He became selfish and vindictive, would kick animals and elbow passers by. Periods of inertia alternated with repetitive walking or reckless driving expeditions. He became a food fadist. He was admitted ultimately to hospital care because of his unpredictable aggression. Although he was uncommunicative, he remained independent in mobility, eating, and dressing. Throughout his illness there was no evidence of disordered perceptual or spatial skills and no abnormal neurological signs were recorded. Unexplained episodes of choking and vomiting recurred in his last 2 years, when he became incontinent. He died of bronchopneumonia 9 years after onset of language disorder, aged 71 years.

\section{Case 2}

A right handed distribution manager began, at the age of 63 , to make phonemic and transpositional errors in speech-for example, "par carp" instead of "car park". There were no semantic paraphasias or grammatical errors and his comprehension was unaffected. Within months, highly uncharacteristic behaviour occurred. He became thoughtless, uncaring, and apathetic, and either inertly 
Figure 1 SPECT scans from cases 1 and 2, showing respectively (a) markedly asymmetric reduction in uptake of tracer affecting the left hemisphere and (b) bilateral reductions in uptake in the anterior hemispheres more marked on the right.

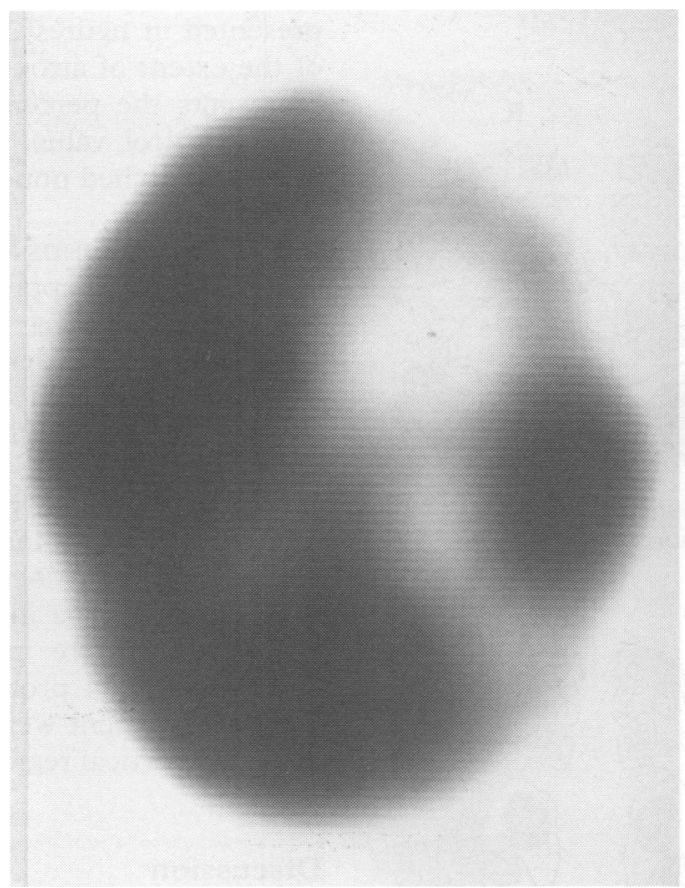

watched television or wandered the neighbourhood without ever becoming lost. He began to drive recklessly. As his expressive difficulties progressed, he appeared unaware of his spoken errors. In addition, he became increasingly callous and aggressive and there were episodes of incontinence for which he showed no concern. His altered behaviour resulted in a psychiatric referral 3 years after onset of symptoms. He was noted at that time to have difficulties in verbal expression and his speech output was non-fluent, broken, and contained literal paraphasic errors. There was word finding difficulty. However, comprehension was relatively intact. Moreover, he demonstrated good day to day memorising and preserved knowledge of current events. He could assimilate a short story and recall it accurately after a delay.

Two years later he was institutionalised because of his aggression. He had become increasingly belligerent, would kick children and elbow passers by. He neglected personal hygiene and table manners, and was frequently incontinent. He engaged in no purposeful activity. Despite his behaviour he was physically well and neurological examination was unremarkable. Neuropsychological evaluation demonstrated a marked language disorder. Speech was non-fluent, effortful, and telegrammatic in style, with omission of grammatical filler words. There were frequent literal but no verbal paraphasias. Occasional palilalia occurred. His understanding of individual word semantics was good, but he was unable to understand complex syntactic sentences. His repetition span was limited to three digits and a single word only. Reading of single words contained literal paralexias and was occasionally palilalic. Writing to dictation was telegraphic: he omitted functional words, and execution was markedly perseverative. There was no evidence of disordered visual perception or spatial functioning and

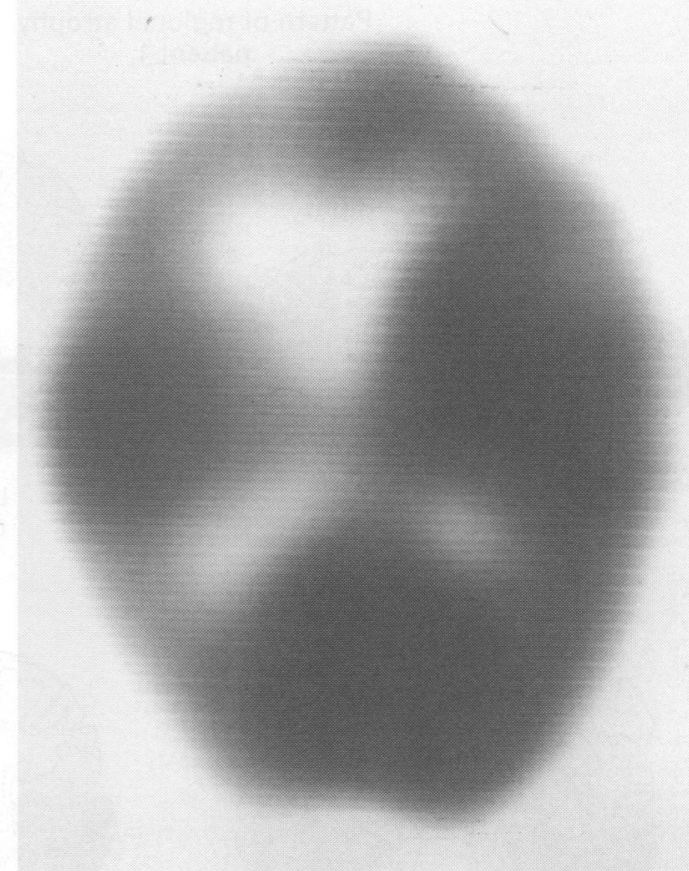

his drawings showed preserved spatial configuration, despite some perseverative pencil strokes. Indeed, he was observed to play pool, dress himself, and find his way without difficulty. During testing he was distractible, restless, uncompliant, and lacked insight.

Speech became monosyllabic and mutism ensued 6 years after onset of language difficulties. Further behavioural deterioration occurred with aggression, poor compliance, and incontinence. He was observed to seek and steal sweet foods and drinks. In the later stages of his illness he developed unexplained episodes of vomiting and he died of bronchopneumonia 8 years after onset of language symptoms, aged 71 years.

\section{Single photon emission computerised tomography}

SPECT scans of the two brothers, carried out respectively 9 and 6 years after the onset of language symptoms are shown in figure 1 . The scan of case 1 showed reduced uptake of tracer extending throughout the left hemisphere. The scan of case 2 showed reduced uptake bilaterally in the anterior hemispheres, more marked on the right.

\section{Pathological analysis}

GROSS APPEARANCES

In case 1 there was markedly asymmetric distribution of atrophy, gross on the left side affecting particularly frontal, temporal, frontoparietal, and lateral parietal/occipital regions. In the right hemisphere severe atrophy was restricted to the anterior temporal lobe. In case 2 there was severe atrophy predominantly of both frontal and temporal lobes, more marked on the right than the left.

Morphological data for the two patients illustrating the topographical distribution of pathological change in the two patients are 
Pattern of regional atrophy patient 1

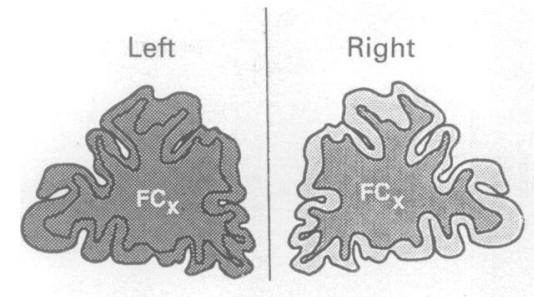

Frontal lobe

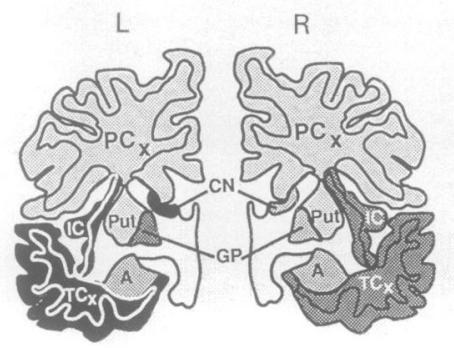

Midpoint of the amygdala A

Pattern of regional atrophy

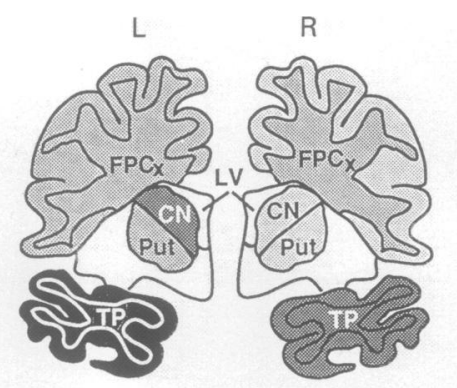

Level of head of caudate nucleus

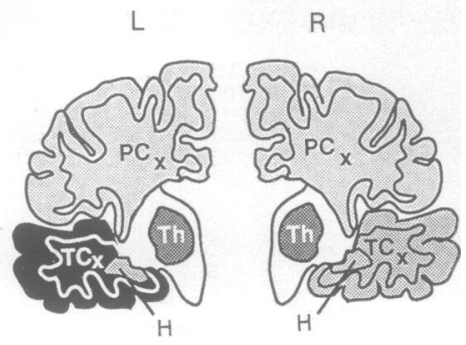

Level of midpoint of the hippocampus patient 2

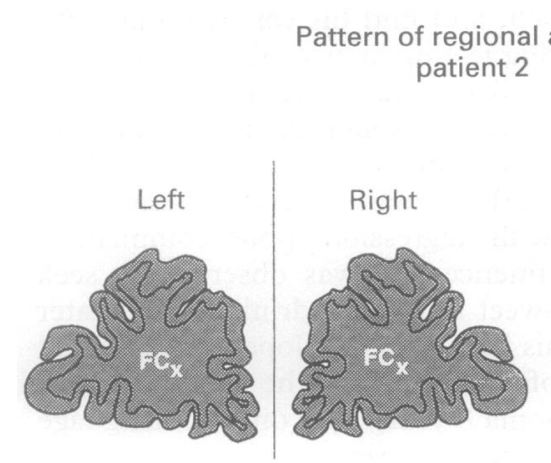

Frontal lobe

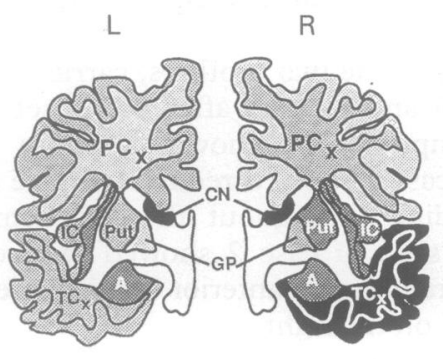

Midpoint of the amygdala

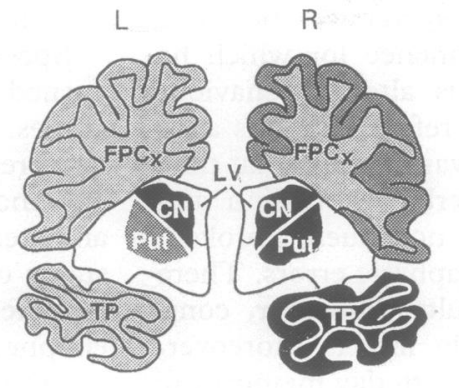

Level of head of caudate nucleus

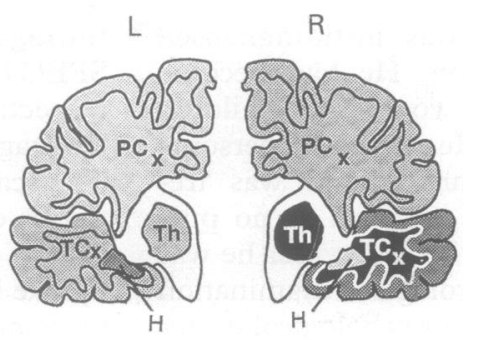

Level of midpoint of the hippocampus

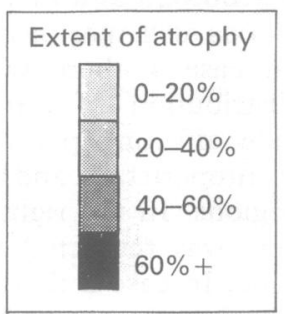

Figure 2a Topographical distribution of cerebral atrophy for case 1. Morphometric measures represent percentage decrement from control values. $2 b$ Topographical distribution of cerebral atrophy for case 2. Morphometric measures represent percentage decrement from control values.

presented in figures $2 a$ and $2 b$. The measure of the extent of atrophy for each brain region represents the percentage decrement from a mean control value, derived from brains of five age-matched non-demented males.

\section{HISTOLOGICAL FINDINGS}

The histological appearance of the neocortex and other regions in case 1 has been described by us in detail previously. ${ }^{1}$ In case 2 the histopathological findings in the neocortex were essentially the same, consisting of a severe loss of large cortical nerve cells, a spongiform change principally affecting the outer cortical laminae and a mild gliosis, mostly subpial in location. No swollen cells, Pick or Lewy-type inclusions, or neurofibrillary tangles were present. A few diffuse deposits of $\beta / \mathrm{A} 4$ protein, consistent with the age of the patient were observed especially in posterior cortical regions.

\section{Discussion}

The two brothers showed a similar progressive aphasic disorder but differed in the relative onset and severity of behavioural disturbance. The pattern of language disorder in both was of a non-fluent aphasia, with phonemic paraphasias, leading to increasingly telegrammatic speech and eventually mutism. Both patients developed behavioural changes during the course of their illness, sharing the characteristics of DFT. Nevertheless, in case 1 behavioural changes developed very late in the course of disease, 7 years after onset of language symptoms. In contrast, in case 2 behavioural changes became apparent and severe almost immediately after the onset of language symptoms. These differences suggest the emergence of "frontal lobe" dysfunction early in the course in case 2 , but late in the case of case 1 .

The clinical differences are reflected in differences in SPECT images. The highly asymmetric appearance, involving the left hemisphere, seen in case 1 is consistent with that reported in other patients with slowly progressive aphasia. The marked bilateral anterior hemisphere abnormality in case 2 is, in contrast, with that of patients with DFT.

The brothers' brains share a similar nonAlzheimer's disease pathology, identified as frontal lobe degeneration by Brun. ${ }^{4}$ However, the distribution of pathological change differs, and reflects the clinical differences and SPECT findings. The extensive atrophy of the left hemisphere seen in case 1 has been recorded in other patients with progressive aphasia. Yet, atrophy in case 1 was not confined to the left hemisphere: severe but restricted atrophy of the right anterior temporal lobe also occurred. In case 2, the marked atrophy of frontotemporal regions bilaterally is similar to the distribution of pathology seen in DFT, ${ }^{2}$ and also in DFT with motor neuron disease. ${ }^{3}$ The greater emphasis of pathology in the right hemisphere is surprising given his clinical presentation with progressive aphasia and, presumably, underlines the vulnerability 
of language to relatively small brain lesions, and explains why lobar atrophies of the left hemisphere are recorded with greater frequency than those of the right. In any event, the findings highlight the association, both behaviourally and pathologically, between progressive aphasia and DFT, and lend support to the view that in lobar atrophies of non-Alzheimer's disease type the particular neuropsychological syndrome is determined by the distribution of pathological changes. The high familial incidence in both progressive aphasia, ${ }^{1}$ and $\mathrm{DFT}^{56}$ and DFT with motor neuron disease ${ }^{3}$ provides further support for their association.

The histology of gliosis, Pick inclusion bodies, and Pick cells (Pick's disease) did not occur in these two cases but has been reported in a proportion of patients with progressive aphasia, ${ }^{7-9}$ DFT, ${ }^{24}$ and DFT and motor neuron disease. ${ }^{1011}$ Other patients have the milder spongiform pathology of frontal lobe dysfunction. ${ }^{4}$ This suggests that the histology represents a spectrum of change in brains with frontotemporal atrophy and may reflect a common underlying molecular biological process which remains to be determined.
We thank Dr HJ Testa for providing SPECT analysis.

1 Snowden JS, Neary D, Mann DMA, et al. Progressive language disorder due to lobar atrophy. Ann Neurol 1992;31:174-83.

2 Mann DMA, South PW, Snowden JS, Neary D Dementia of frontal lobe type; neuropathology and Dementia of frontal lobe type; neuropathology and
immunohistochemistry. $f$ Neurol Neurosurg Psychiatry 1993;56:605-14.

3 Neary D, Snowden JS, Mann DMA, et al. Frontal lobe dementia and motor neuron disease. $\mathcal{F}$ Neurol Neurosurg Psychiatry 1990;53:23-32.

4 Brun A. Frontal lobe degeneration of non-Alzheimer type. I. Neuropathology. Arch Gerontol Geriatr 1987;6: 193-207.

5 Gustafson L. Frontal lobe degeneration of non-Alzheimer type. II. Clinical picture and differential diagnosis. Arch Gerontol Geriatr 1987;6:209-23.

6 Neary D, Snowden JS, Northen B, Goulding PJ. Dementia of frontal lobe type. I Neurol Neurosurg Psychiatry 1988;51:353-61.

7 Wechsler AF, Verity A, Rosenschein S, et al. Pick's disease. A clinical, computed tomographic and histologic study with Golgi impregnation observations. Arch study with Golgi improl $1982 ; 39: 287-90$.

8 Holland AL, McBurney DH, Moossy J, Reinmuth OM. The dissolution of language in Pick's disease with neurofibrillary tangles: a case study. Brain Lang 1985; 24:36-58.

9 Graff-Radford NR, Damasio AR, Hyman BT, et al. Progressive aphasia in a patient with Pick's disease: a neuropsychological, radiologic, and anatomic study. Neurology 1990;40:620-6.

10 Brion S, Psimaras A, Chevalier JF, et al. L'association maladie de Pick et sclerose laterale amyotrophique. Etude d'un cas anatomo-clinique et revue de la litterature. L'Encephale 1980;6:250-86.

11 Constantinidis J. Syndrome familial: association de maladie de Pick et sclerose laterale amyotrophique. L'Encephale 1987;13:285-93. 